\title{
Adeno-Associated Virus Transfer of a Gene Encoding SNAP-25 Resistant to Botulinum Toxin A Attenuates Neuromuscular Paralysis Associated with Botulism
}

\author{
Arvind Raghunath, ${ }^{1}$ Francesc Perez-Branguli, ${ }^{1}$ Leonard Smith, ${ }^{2}$ and J. Oliver Dolly ${ }^{1}$ \\ ${ }^{1}$ International Centre for Neurotherapeutics, Dublin City University, Dublin 9, Ireland, and ${ }^{2}$ Integrated Toxicology Division, United States Army Medical \\ Research Institute of Infectious Diseases, Fort Detrick, Maryland 21702-5011
}

\begin{abstract}
Advances in viral gene therapy have opened new possibilities for treating a range of motor neuron diseases, but these have not yet been translated into clinically applicable therapies because of difficulties in delivery to susceptible/damaged neurons, ambiguities in the identity of gene(s) implicated, and a paucity of means to quantify any physiological improvement. Most of these hurdles can be overcome by using the neuromuscular paralysis induced by botulinum neurotoxin type A (BoNT/A) as a prototype disease. Furthermore, because human botulism, occasionally fatal, causes prolonged muscle disablement as a result of the intraneuronal persistence of the toxin's SNAP-25 (S25)-cleaving protease, development of a genetic approach could lead to a potential treatment for this debilitating disease. Adeno-associated viral delivery of a cleavage-resistant S25 gene (S25-R198T) to chromaffin cells in vitro yielded exocytotically active S25-R198T that diminished subsequent blockade by BoNT/A of evoked catecholamine release. Evaluation in vivo, by administering this virus into rat spinal cord before injecting BoNT/A, showed a decreased inhibition of acetylcholine release as reflected in elevated retention of neuromuscular transmission. A similar, although smaller, protection of synaptic transmission from the toxin was seen after peripherally injecting the therapeutic virus. Such therapy also curtailed nerve sprouting normally induced by BoNT/A. This first demonstration of the utility of a DNA-based therapy for botulism paves the way for further advances in its treatment and for application to genetic disorders of motor neurons.
\end{abstract}

Key words: botulinum; gene transfer; neuromuscular junction; motor neuron; SNARE proteins; sprouting

\section{Introduction}

A major challenge facing gene therapy for motor neuron disorders, a group of rare yet debilitating and ultimately fatal diseases affecting spinal motor neurons, is targeted, minimally traumatic delivery. Because of the limited number of motor neurons (e.g., rat sciatic nerve has only $\sim 2000$ efferents) (NicolopoulosStournaras and Iles, 1983), insults that are merely sublethal in other cells can be extremely deleterious [e.g., in superoxidedismutase mutant mice, which ubiquitously express the damaged protein, motor neurons are the most affected (Bruijn et al., 2004)]. Their anatomical structure, with a relatively inaccessible cell body in the spinal column and an elongated axon, complicates therapies including gene transfer by viral vectors. Those that transfect motor neurons [adeno-associated virus (AAV), lentivirus, and herpes virus] do so most efficiently when in direct contact with the cell body (Mannes et al., 1998). Because intraspinal injection is difficult under clinical conditions, the use of periph-

Received Nov. 15, 2007; revised Feb. 7, 2008; accepted Feb. 8, 2008.

This work was supported by United States Army Medical Research Institute of Infectious Diseases Contract DAMD17-03-C-0094 and Defense Threat Reduction Agency-Joint Science and Technology Office, Medical Science and Technology Division Contract HDTRA-1-07-C-0034.

Correspondence should be addressed to Prof. J. Oliver Dolly, X230, International Centre for Neurotherapeutics, Dublin City University, Glasnevin, Dublin 9, Ireland. E-mail: oliver.dolly@dcu.ie.

DOI:10.1523/JNEUROSCI.5690-07.2008

Copyright $\odot 2008$ Society for Neuroscience $\quad$ 0270-6474/08/283683-06\$15.00/0 erally deliverable vectors would be desirable; moreover, such an advance requires a model that allows accurate measurement of the graded improvements currently achievable.

An ideal system to evaluate such therapies is the blockade of neuromuscular transmission by botulinum neurotoxin type $\mathrm{A}$ (BoNT/A). Its light chain $\mathrm{Zn}^{2+}$-dependent endoprotease causes irreversible blockade of acetylcholine (ACh) exocytosis by specific cleavage of SNAP-25 (S25), a protein essential for this fundamental process (Blasi et al., 1994). Importantly, protection from inhibition by BoNT/A can be achieved by expression of a toxin-resistant S25 mutant gene (O'Sullivan et al., 1999). Furthermore, because current treatments are ineffective in combating botulism, it is imperative that a refined therapy is designed with the ability to abrogate the prolonged convalescence and high mortality and morbidity associated with this condition.

Herein, sustained neuromuscular paralysis by BoNT/A was attenuated using AAV vectors that give long-term expression of a cleavage-resistant S25 mutant (S25-R198T), having all the properties of the wild type, including ability to substitute for truncated S25 and recover exocytosis in intoxicated chromaffin cells (O'Sullivan et al., 1999). AAV vectors provide an efficient and relatively safe way to ensure prolonged expression of genes in neurons (Burger et al., 2005), and botulism in rats provides a much more valid model for evaluating this gene therapy. Injection of AAVs encoding S25-R198T into the spinal anterior horn 
innervating the soleus muscle protected neuromuscular transmission from subsequent inhibition by BoNT/A. Moreover, muscles treated in this way showed a decreased amount of nerve sprouting compared with unprotected controls. Finally, administering the therapeutic AAVs directly into the soleus muscle resulted in their retrograde transport to motor cell bodies and a consequential reduction of BoNT/A-mediated blockade of neurotransmission at peripheral synapses. These findings provide the first evidence for the potential applicability of gene therapy to the intractable problems posed by botulism and, possibly in the longer-term, genetic disorders of motor neurons.

\section{Materials and Methods}

Construction, production, and purification of recombinant $A A V$ s expressing BoNT-resistant S25. PCR, using the S25BamH1His ${ }_{6}$-For and S25XhoIRev primer pair, generated a $\mathrm{His}_{6}$-tagged S25 from a mouse S25 gene; this was inserted into the pAAV-MCS plasmid to generate the pAAV-His ${ }_{6}$ S25 plasmid [ $\sim 96 \%$ homologous to human S25 (for primer sequences, see supplemental material, available at www.jneurosci.org)]. A similar technique, with the substitution of S25RTXhoI-Rev for S25XhoI-Rev, created pAAV-His ${ }_{6}-\mathrm{S} 25-\mathrm{R} 198 \mathrm{~T}$ encoding a BoNT/A-resistant S25. These different plasmids, along with AAV-hrGFP, were used to create their respective replication-deficient AAVs and purified on heparin-agarose, as described previously (Zolotukhin et al., 1999). Viral (protein) yield and purity were assessed on Coomassie-stained SDS-PAGE gels by comparing the surface proteins (VP1, VP2, and VP3) to a bovine serum albumin (BSA) standard. Real-time quantitative PCR, with appropriate primers, quantified the number of genomic copies (GCs) per preparation, and a dilution series applied to HEK-293 cells yielded the number of transducing units.

Culture of chromaffin cells, infection with recombinant $A A V$ s, intoxication with BoNT/A, and assay of catecholamine release. Bovine adrenal chromaffin cells were isolated as described previously (Lawrence et al., 1996), infected with AAVs, and $7 \mathrm{~d}$ later exposed for $24 \mathrm{~h}$ to $10 \mathrm{~nm}$ BoNT/A under conditions that promote its uptake. $\mathrm{Ba}^{2+}$-evoked catecholamine release was measured after different viral treatments and normalized to that in the presence of the toxin alone and presented as mean $\pm \mathrm{SD}(n=4)$, from two or more sets of experiments. $t$ tests were used to determine the statistical significance.

Stereotaxic intraspinal administration of $A A V$ s for transfection of anterior horn neurons supplying the soleus muscle. Rats were anesthetized with medetomidine/fentanyl citrate, and the T10-L2 vertebrae were exposed and positioned in a stereotaxic frame using spinal adapters (Stoelting, Wood Dale, IL). After careful penetration of the vertebral lamina over the T13 spinal level using a microdrill, known quantities of the virus were injected over 5 min with a Hamilton microsyringe attached to a $30 \mathrm{ga}$ beveled needle. After a further $5 \mathrm{~min}$, the needle was withdrawn and the procedure repeated at L1 and L2 levels; twitching of the lower limb muscles confirmed the accurate placement of the needle. The paraspinal muscles and the superficial fascia, reinforced with a polypropylene mesh, were sutured with vicryl sutures, and the skin was closed with Prolene sutures before injection of atipamazole/nalbuphine and carprofen/bupivacaine.

In situ recording of neuromuscular transmission after BoNT/A administration. Three weeks after the intraspinal injection, $\sim 1$ mouse $\mathrm{LD}_{50}$ of BoNT/A in $10 \mu \mathrm{l}$ of normal saline (NS)/BSA was injected into the soleus muscle. After a $16-25 \mathrm{~d}$ recovery, the rats were anesthetized, and the soleal contribution to the Achilles tendon was isolated and ligated to a sensitive force transducer (FORT10 and PowerLab 4/20T; ADInstruments, Castle Hill, New South Wales, Australia). The sciatic nerve, exposed at midthigh level, and the belly of the soleus muscle were stimulated at $1 \mathrm{~Hz} / 200 \mathrm{mV} / 0.2 \mathrm{~ms}$ and $1 \mathrm{~Hz} / 5 \mathrm{~V} / 2 \mathrm{~ms}$, respectively; $60 \mathrm{~Hz}$ stimulation provided the response at tetanic frequencies.

Immunohistochemical detection of S25, $\mathrm{His}_{6}-\mathrm{S} 25, \mathrm{His}_{6}-\mathrm{S} 25-\mathrm{R} 198 \mathrm{~T}$, and GFP expression. Serial $25 \mu \mathrm{m}$ muscle and spinal cord sections were permeabilized before exposure to the primary antibody (1:50 for anti-NF200 and anti-His ${ }_{6}$ ) diluted in PBS with $0.05 \%$ Tween $20 / 1 \%$ BSA/2\% goat serum and, after washing, to the relevant secondary antibodies $(1: 50$
Alexa-488 or 1:100 A546/phycoerythrin-labeled goat anti-mouse) with or without 1:500 fluorescently tagged $\alpha$-bungarotoxin ( $\alpha$-BuTx; with Alexa 488 or rhodamine). These were visualized with an Olympus (Tokyo, Japan) IX51 inverted fluorescent microscope or, when appropriate, a Zeiss (Thornwood, NY) confocal microscope (LSM510).

Determination of the distribution of ACh receptors at neuromuscular junctions using fluorophore-conjugated $\alpha$-BuTx. Random soleal sections from rats injected with BoNT/A alone, AAV-His ${ }_{6}-\mathrm{S} 25-\mathrm{R} 198 \mathrm{~T}$, and then BoNT/A and untreated controls were stained with an $\alpha$-BuTx-Alexa- 488 conjugate; for determination of muscle parameters, see supplemental material (available at www.jneurosci.org). Staining with anti-NF200 and fluorescently tagged $\alpha$-BuTx allowed visual monitoring of sprouting at neuromuscular junctions (NMJs) protected by viral-mediated production of BoNT/A-resistant S25.

\section{Results}

\section{Protection of stimulated exocytosis from BoNT/A-induced blockade by prior expression of $\mathrm{His}_{6}-\mathrm{S} 25-\mathrm{R} 198 \mathrm{~T}$, but not wild-type $\mathrm{S} 25$, in chromaffin cells}

AAV helper-free vector system based on a nonpathogenic parvovirus was used to construct recombinant type-2 AAVs incorporating different genes. Initially, control viruses expressing the hrGFP marker protein were produced; heparin affinity chromatography yielded pure viruses containing only viral surface proteins (Fig. $1 A, B$ ). Their activity was established from expression of hrGFP fluorescence in infected chromaffin cells (Fig. 1C). Later, wild-type or mutated S25, engineered to be nonsusceptible to cleavage by BoNT/A, were introduced into this vector. In some cases, the proteins were coexpressed with an hrGFP marker using an IRES segment (AAV-His 6 -S25-R198T-IRES-hrGFP); coexpression was demonstrated by immunodetection of $\mathrm{His}_{6}, \mathrm{~S} 25$, and hrGFP in lysed cells (Fig. $1 D$ ).

Their protective abilities were demonstrated in chromaffin cells exposed for $7 \mathrm{~d}$ to various concentrations of the recombinant viruses before challenge with $10 \mathrm{~nm}$ BoNT/A (the $150 \mathrm{kDa}$ neurotoxin free of accessory proteins was used throughout). AAV-His ${ }_{6}$-S25-R198T viruses gave a dose-dependent relative increase in exocytosis of $\sim 55 \%$ compared with the level recorded for cells treated with toxin alone (Fig. $1 E$ ). The highest concentration of viral particles tested, equivalent to a multiplicity of infection $=50$, infected $\sim 90 \%$ of the cells. The AAV-His ${ }_{6}-\mathrm{S} 25$ R198T-IRES-hrGFP virus yielded a lower, yet significant, relative protection $(\sim 23 \%)$, whereas overexpression of wild-type S25 failed to show any effect (Fig. $1 E$ ). This extent of prophylaxis, despite using large numbers $\left(>10^{6}\right.$ cells per well) of postmitotic neuroendocrine cells, justified progression to in vivo testing of this gene therapy paradigm. Moreover, infection of chromaffin cells with viral particles expressing toxin-resistant, but not wildtype, S25 gave appreciable rescue of evoked catecholamine exocytosis that had been inhibited by preexposure to BoNT/A (see supplemental material, available at www.jneurosci.org).

\section{Efficient expression of cleavage-resistant S25 in peripheral nerve terminals of rats after intraspinal administration of AAVs protects against the neuroparalytic effects of BoNT/A} Published data (Adler et al., 2001) determined the choice of rat soleus muscle as an in vivo system to study the effects of viral vector-induced BoNT prophylaxis. The efficiency of neuromuscular transmission (ENT; the ratio of the force of contraction elicited by nerve stimulation to that elicited by direct muscle stimulation) provides an accurate and sensitive means to determine the progress of, and recovery from, toxin-induced paralysis. Stereotaxic injection of AAVs expressing marker hrGFP and/or $\mathrm{His}_{6}-\mathrm{S} 25-\mathrm{R} 198 \mathrm{~T}$ into the right anterior T12, L1, and L3 spinal 
A

C

M Lys De Ft1 E M Ft2 Conc

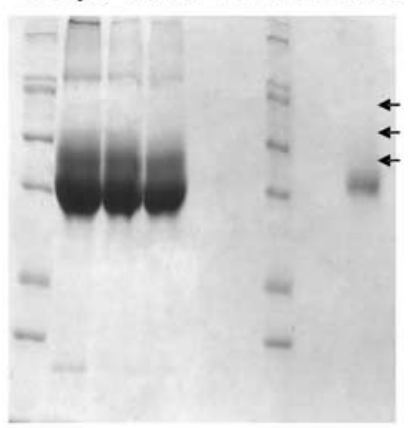

B

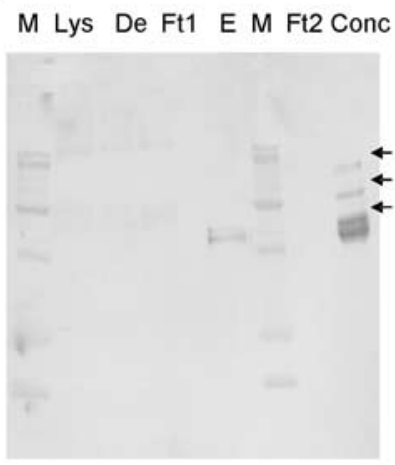

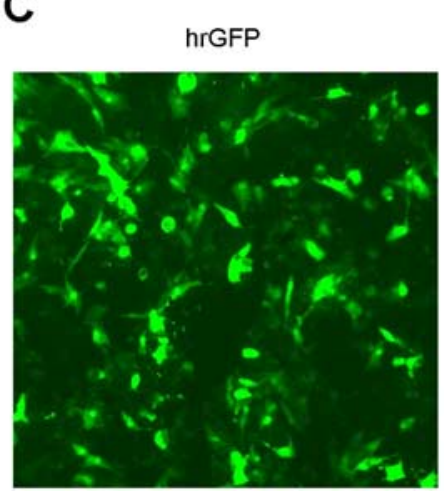

D

(i)

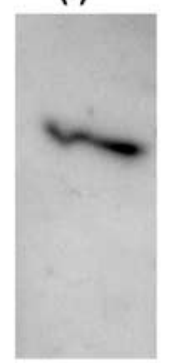

a-hrGFP

(ii)

(iii)

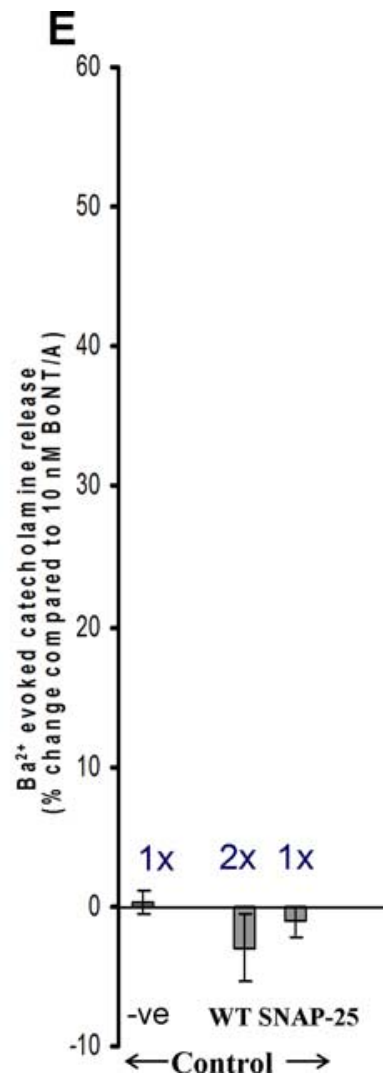

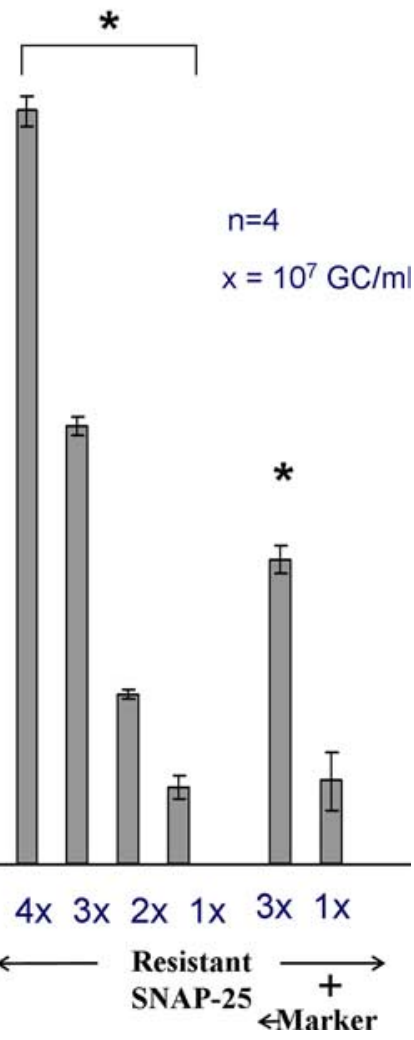

Figure 1. AAV-mediated expression in chromaffin cells of cleavage-resistant $S 25$, but not wild-type, antagonizes inhibition of catecholamine release by BoNT/A. $\boldsymbol{A}, \boldsymbol{B}, \mathrm{Coomassie-stained} \mathrm{gel}(\boldsymbol{A})$ and Western blot $(\boldsymbol{B})$ of aliquots from stages of the production of AAV-hrGFP. M, Markers; Lys, lysate; De, deoxycholate extract; Ft1, flow-through from heparin-agarose column; $E$, eluate; Ft2, flow-through from concentrator; Conc, concentrated AAV-hrGFP. Arrows show surface proteins VP1, VP2, and VP3 with $M_{\mathrm{r}}=87,000,73,000$, and 62,000, respectively. C, Fluorescent microscopy

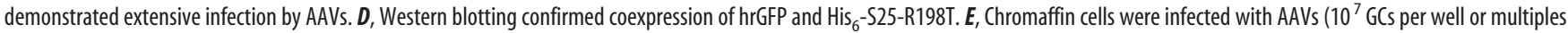
expressing $\mathrm{His}_{6}$-S25WT, $\mathrm{His}_{6}$-S25-R198T, or His ${ }_{6}$-S25-R198T plus hrGFP) or vehicle and $7 \mathrm{~d}$ later exposed to $10 \mathrm{~nm}$ BoNT for $24 \mathrm{~h}$ before quantifying Ba ${ }^{2+}$-evoked catecholamine release. Averaged relative release from virus-treated and control cells \pm SD is plotted $(n=4)$. Cells expressing cleavage-resistant $S 25$ showed a significant increase in catecholamine release $\left({ }^{*} p<0.01\right.$, as determined by a $t$ test) compared with controls.

horns, containing the cell bodies of neurons supplying the soleus, resulted in a localized coexpression of hrGFP and $\mathrm{His}_{6}-\mathrm{S} 25$ R198T, confirming the accuracy of the microsurgical procedure (Fig. 2A). After 6-7 weeks, the expressed proteins were also seen in the periphery at NMJs, identified by colocalization with $\alpha$-BuTx (hrGFP and $\alpha$-BuTx in Fig. 2 B and $\mathrm{His}_{6}$-S25-R198T and $\alpha$-BuTx in Fig. 2C). The functional effectiveness of this cleavageresistant S25 was established by bilateral injection of $\sim 1$ mouse $\mathrm{LD}_{50}$ of BoNT/A in NS/BSA into the soleus $21 \mathrm{~d}$ after viral injection, followed by ENT measurements after a further $16 \mathrm{~d}$. The expected reduction in transmitter release after injecting BoNT/A alone was confirmed, whereas the maintenance of near-normal neurotransmission in synaptic junctions overexpressing hrGFP or S25-R198T demonstrates purity of the preparations and the minimal trauma caused by the stereotaxic technique (Fig. $2 \mathrm{Di}$, Dii). Animals preinjected with AAV$\mathrm{His}_{6}$ S25-R198T showed a significantly greater retention of neuromuscular transmission (Fig. 2 Div) compared with the unprotected (contralateral) side after a BoNT/A challenge (Fig. 2 Diii) $(p<0.05)$; summarized data from four animals for each group are depicted in Figure $2 E$. This attenuation of BoNT/A-induced neuroparalysis is the first ever demonstration of a nonimmunological protective therapy in whole animals and establishes the functional effectiveness of the peripherally delivered cleavage-resistant S25.
BoNT/A-induced synapse remodeling is reduced by protection of neuromuscular transmission with a spinal injection of $\mathrm{AAV}-\mathrm{His}_{\mathbf{6}}-\mathrm{S} 25-\mathrm{R} 198 \mathrm{~T}$

Sections of soleal muscles from BoNT/A-treated and control rats were stained with $\alpha$-BuTx-A488 and the anti-NF200 antibody to visualize the postsynaptic and presynaptic parts of the NMJ, respectively. Comparison of images from control and those treated 16 or $25 \mathrm{~d}$ earlier with BoNT/A revealed a much greater number of sprouts in the latter (Fig. 3, compare $A$ with $B-F$ ). Additionally, in agreement with published results (Brown et al., 1980), it was observed that axonal and endplate sprouting frequently coexisted in the same muscle and, sometimes, in the same endplate (Fig. 3F). After characterizing BoNT/A-induced sprouting, the effect of protection on this process was determined. Immunostaining of soleal sections treated with toxin for $16 \mathrm{~d}$ showed less extensive sprouting at NMJs of rats that received BoNT/Aresistant S25 than those given toxin alone (Fig. 3G,H). To quantify these changes, the amount of sprouting in "protected (by AAV-His ${ }_{6}$-S25-R198T) and challenged (by BoNT/A)" muscles were compared with "unprotected and challenged" or "unprotected and unchallenged" muscles. Because changes in ACh receptor density and distribution are highly sensitive to perturbations of synaptic transmission, an automated quantitation of $\alpha$-BuTx-fluorophore conjugate binding to the postsynaptic part of the NMJ was chosen (Pestronk and Drachman, 1978; Wang et 
A

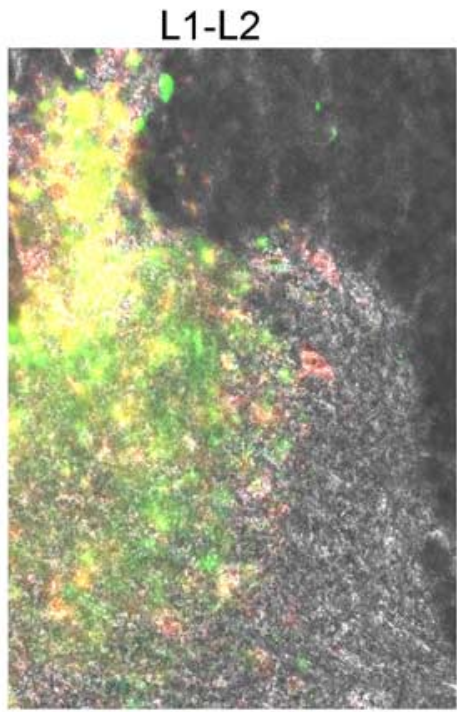

L3-L4

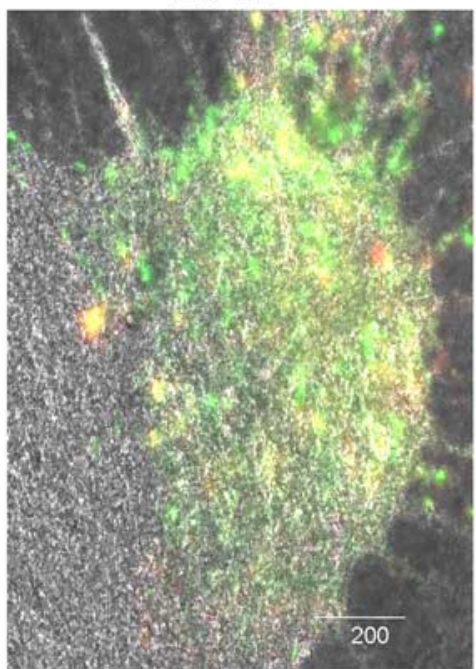

B

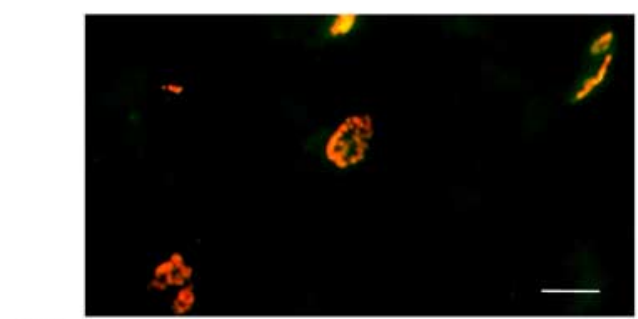

C

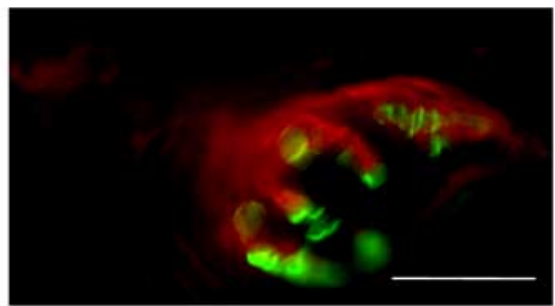

D

(i)

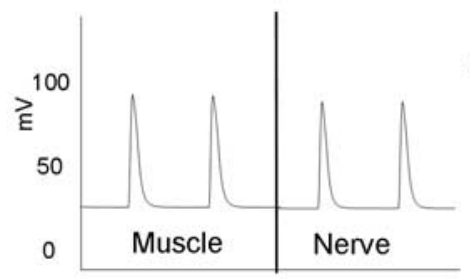

(ii)

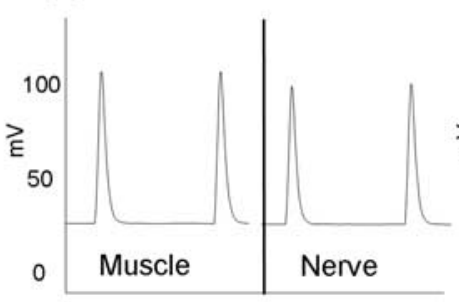

(iii)

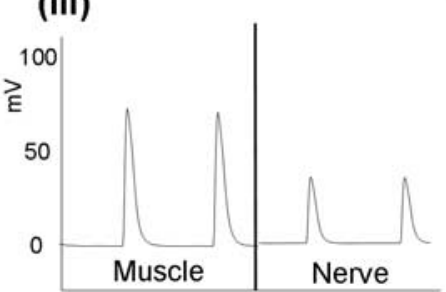

(iv)

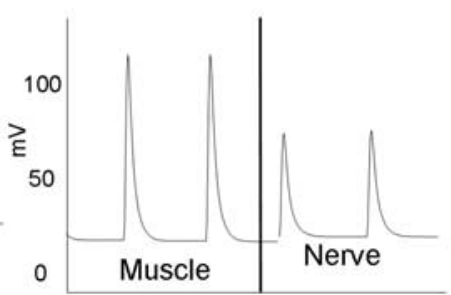

E

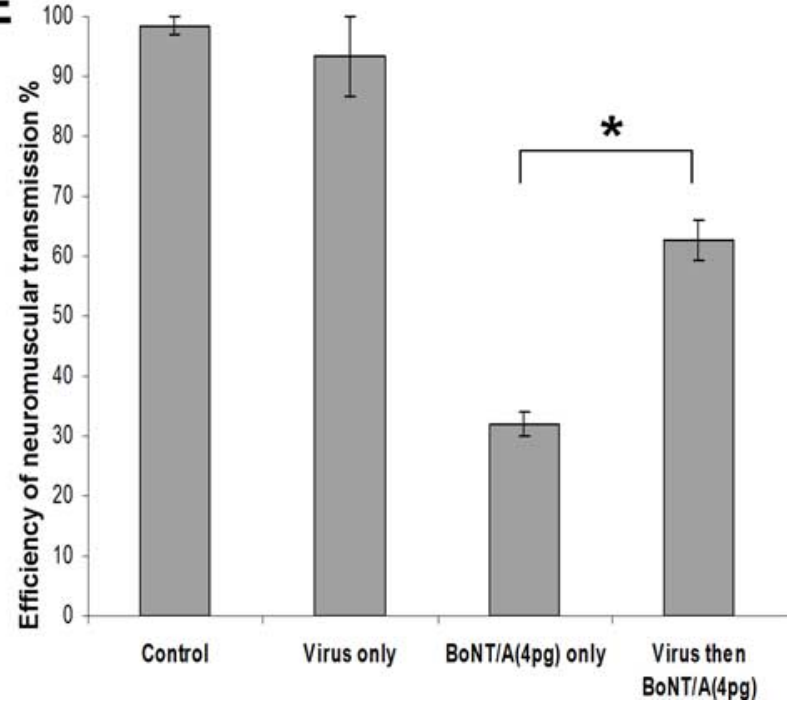

Figure 2. Treatment with AAV-His ${ }_{6}$-S25-R198T protects against neuromuscular paralysis induced by BoNT/A. $A$, Spinal cord sections from rats injected with AAVs (5 $\mu$ l of $10{ }^{11} \mathrm{GC} / \mathrm{ml}$ ) expressing hrGFP (green) and His ${ }_{6}$-S25-R198T (red) show colocalization in the right L1/L2 and L3/L4 anterior horns. Scale bar, $200 \mu \mathrm{m} . \boldsymbol{B}, \boldsymbol{C}$, Delivery of hrGFP (green; $\boldsymbol{B}$ ) and His ${ }_{6}$ S25-R198T (red; $\boldsymbol{C}$ ) to presynaptic terminals demonstrated by colocalization with the postsynaptic $A C h$ receptor ( $\alpha$-BuTx, red in $\boldsymbol{B}$, green in $\boldsymbol{C}$ ), in soleal sections from rats injected with AAV-hrGFP $(\boldsymbol{B})$ or AAV-His ${ }_{6}$-S25R198T (C). Scale bars, $20 \mu \mathrm{m}$. D, Effect on ENT of spinal injection of AAV-hrGFP (i) and AAV-His - -S25-R198T (ii, iv) or vehicle (iii) into animals, some of which were challenged with BoNT/A 21 d later (iii, iv). Representative contractions after a further $16 \mathrm{~d}$ from single supramaximal stimulation of the sciatic nerve (right) and direct stimulation of the muscle (left) are shown. $E$, Summarized data showing that viral pretreatment protects ENT from subsequent paralysis by BoNT/A; each bar represents the mean \pm SD of values from four animals $\left({ }^{*} p<0.05\right)$.

al., 2005). Relative to untreated muscles, significant increases were found after BoNT/A treatment in the mean endplate area, the area of the largest endplates and, probably because of the muscle wasting that accompanies paralysis, the endplate space occupied per muscle section (Fig. 3I). However, interestingly, the average endplate area was significantly reduced in those endplates expressing S25 R198T compared with controls [treated with the toxin alone (Fig. $3 J$ ) ]. A lesser, nonsignificant reduction was seen in the maximum endplate and in the ratio of endplate to muscle areas (Fig. 3J).

Partial protection against BoNT/A-induced neuroparalysis in rats by neuronal expression of $\mathrm{His}_{6}-\mathrm{S} 25-\mathrm{R} 198 \mathrm{~T}$ after peripheral injection of its AAV construct

Although intraspinal AAV injection achieved efficient transduction and protection of motor neurons against BoNT/A, a periph- erally deliverable therapy is still preferable. The control AAVhrGFP virus underwent retrograde transport to the spinal cord, as confirmed by expression of the marker protein in motor neurons of the anterior horn supplying the soleus muscle (Fig. 4A). Having confirmed the utility of such an approach, the ability of AAV-His ${ }_{6}-\mathrm{S} 25-\mathrm{R} 198 \mathrm{~T}$ to protect the motor neurons from BoNT/ A-mediated paralysis was next evaluated by its injection into the soleus muscle, followed a week later by toxin administration; the contralateral muscle was injected with the toxin alone. When tested 3 weeks after the viral injection, the protected NMJs in the best responding animals showed a fourfold to sixfold higher retention of synaptic transmission [ 18.5 vs $3.2 \%$ for single stimulation (Fig. 4 Bi) and 62.5 vs $15.3 \%$ for tetanic stimulation (Fig. $4 \mathrm{Bii})$ ]. The combined results, from six animals for each treatment, showed that the side injected with the virus gave a $>2.5$ fold increased retention of muscle strength in response to tetanic 

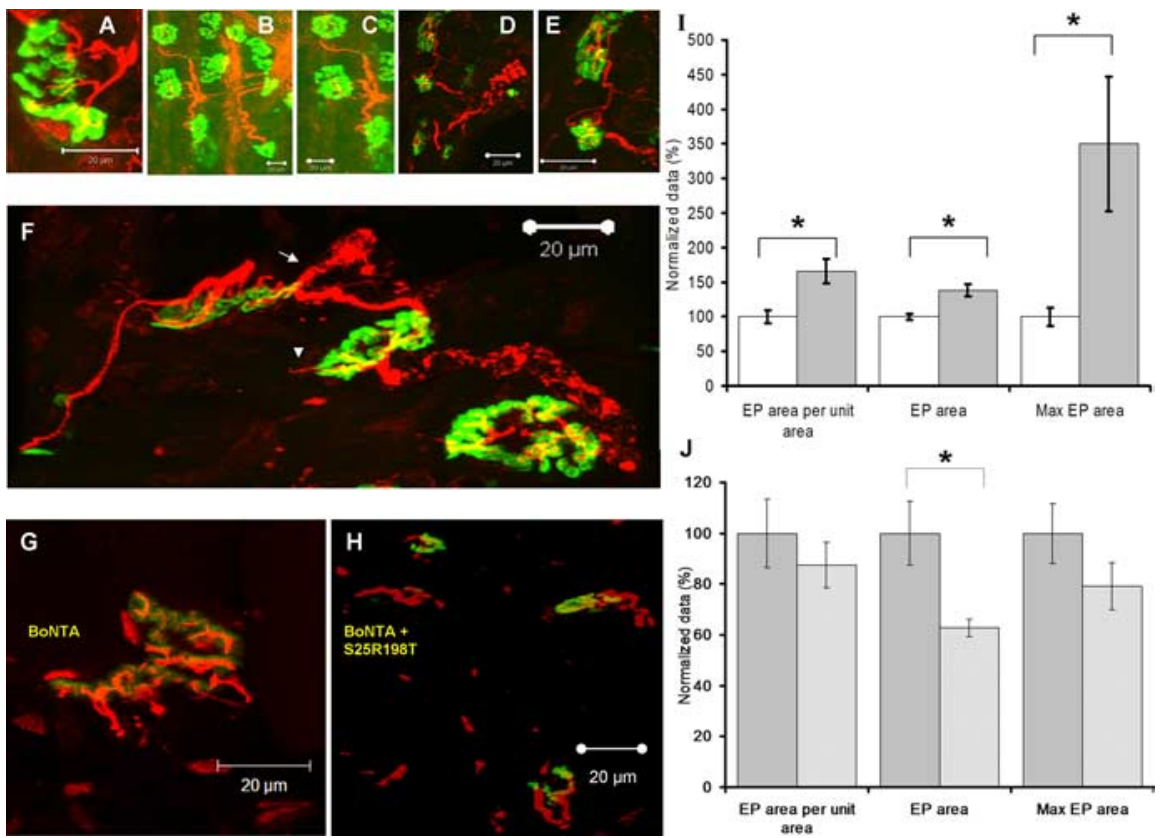

Figure 3. Influence of virally expressed $\mathrm{His}_{6}-\mathrm{S} 25-\mathrm{R} 198 \mathrm{~T}$ on synaptic remodeling in rat soleus muscles induced by BoNT/A. $\boldsymbol{A}-\boldsymbol{F}$, Immunofluorescent images from control $(\boldsymbol{A})$ and muscles injected with $\sim 2 \mathrm{LD}_{50}$ units of $B \mathrm{NT} / \mathrm{A}(\boldsymbol{B}-\boldsymbol{F})$ showing axonal sprouting $(\boldsymbol{B}$, magnified in $\boldsymbol{C})$, terminal sprouting $(\boldsymbol{D}$, magnified in $\boldsymbol{E})$, and mixed sprouting $[\boldsymbol{F}$; axonal sprouting (arrow) and terminal sprouting (arrowhead)]. Scale bars, $20 \mu \mathrm{m}$. $\alpha$-NF200 is shown in red with $\alpha$-BuTx-A488 in green. $\boldsymbol{G}, \boldsymbol{H}$, The effect of having administered AAV-His ${ }_{6}$-S25-R198T 21 d before BoNT/A injection $(\boldsymbol{H})$ compared with the muscle given the toxin only $(\boldsymbol{G}) . \boldsymbol{I}, \boldsymbol{J}, \mathrm{An}$ automated quantitation of sprouting determined the various parameters plotted (as average \pm SEM) in I for muscle treated with BoNT/A (shaded) or vehicle (open) and in J those receiving BoNT/A alone (shaded) or BoNT/A plus AAV-His 6 -S25-R198T (hatched) $\left({ }^{*} p \leq 0.005\right.$ by unpaired $t$ test).

stimulation ( 27.5 vs $10.5 \%$ ) (Fig. $4 B$ ). These investigations provide an encouraging demonstration of the potential of minimally invasive paths to a gene therapy for botulism.

\section{Discussion}

To prevent the prolonged convalescence associated with human botulism caused by BoNT/A, a clinically effective therapy needs to act at multiple stages by antagonizing the toxin's binding or uptake, inhibiting its breakdown of S25, and/or hastening recovery by replenishing truncated S25 with a functional, BoNTresistant form (O’Sullivan et al., 1999). Although progress toward the first two goals is being achieved by pharmacological means using inhibitors of the toxin's protease, a novel gene therapy approach is necessary to attain the final stages, especially in light of the extraordinary persistence of BoNT/A protease at nerve terminals (Keller et al., 1999; Foran et al., 2003; Meunier et al., 2003). The applicability of gene therapy was initially revealed by demonstrating the attenuation of this toxin's inhibition of secretion from chromaffin cells after overexpression of a cleavage-resistant S25 (O’Sullivan et al., 1999). Because the classical gene transfection used in that earlier study gave very low efficiency, much more effective gene delivery is a prerequisite for in vivo therapeutic purposes. To this end, the gene encoding S25-R198T was inserted into an AAV vector yielding a highly efficient, nonpathogenic virus for transferring genes into nondividing neurons.

Initial experiments confirmed that overexpression of a $\mathrm{His}_{6}{ }^{-}$ tagged cleavage-resistant S25 in eukaryotic cells does not overwhelm the posttranslational processes, especially palmitoylation, as revealed by the membrane association of this protein (see supplemental results, available at www.jneurosci.org as supplemental material). This is important in light of the failure of free cytoplasmic S25 to take part in productive exocytosis (Washbourne et al., 2001) and because of the necessity for toxin-resistant S25 to antagonize disabled S25 at the membrane (O'Sullivan et al., 1999). The administered viruses infected $90 \%$ of $\sim 10^{6}$ chromaffin cells and produced enough BoNT/A-resistant S25 to overcome the toxin's inhibitory effects.

Although the in vitro results validated this therapeutic strategy, it was necessary to establish its applicability to the more complex in vivo system. BoNT/A-induced paralysis can be measured by decrease in force of contraction evoked by stimulation of the sciatic nerve in rat limb muscles (Adler et al., 2001). The synaptic remodeling of rat soleal NMJs after neuroparalysis (Pestronk and Drachman, 1978; Brown et al., 1980) was another reason for choosing this muscle. Direct injection into the spinal anterior horn was used initially because it gives a more intense infection of motor neurons and, consequently, recombinant protein production than intrathecal administration (Mannes et al., 1998). Intraspinal injection of cleavage-resistant S25-expressing AAVs resulted in a localized expression of this protein in the lumbar anterior horns and, subsequently, peripheral transport to their synaptic terminals (Fig. 2). Significantly, the expressed and peripherally transported transgenic protein successfully substituted for native cleavage-susceptible S25, as demonstrated by enhanced retention of nerve-stimulated muscle contractility in preprotected animals later challenged with BoNT/A (Fig. 2), detected by single supramaximal stimuli or tetanic pulses with protection being more marked with the latter. Only AAVs expressing $\mathrm{His}_{6}{ }^{-}$ S25-R198T, and not those that produce hrGFP or wild-type $\mathrm{His}_{6}$ $\mathrm{S} 25$, proved effective. This is the first report on successful antagonism of BoNT/A action in live animals using viral-mediated gene delivery. The protection seen demonstrates that the recombinantly produced protein cannot only overcome the persistent protease activity of the toxin at motor neuron terminals, but also any probable inhibition by the truncated native S25 (Dolly and Aoki, 2006). A combination of such gene delivery with effective inhibitors of the protease should help provide both immediate and prolonged therapy.

Published data suggested that AAVs undergo successful retrograde transport from mouse NMJs and can efficiently infect the supplying motor neurons, with $\sim 1$ in 1000 reaching the cell body (Kaspar et al., 2003). Although injection of $>5 \times 10^{11}$ AAVs into soleal muscle proved necessary to detect infected motor neurons, this mode of administration offers advantages of minimal trauma and faster recovery from the operating procedure; most notably, in terms of possible clinical application, it allows easier access to important muscles. The potential of peripheral application is reflected by the promising protection obtained.

It is believed that synaptic remodeling after BoNT/A-induced paralysis plays an important role in the early recovery (de Paiva et al., 1999; Meunier et al., 2003). The quantity of sprouts is thought to depend strongly on the amount of toxin, the kind of muscle [fast muscles have lower amounts of mostly axonal sprouting, compared with slow muscles, which show a higher percentage of 
A

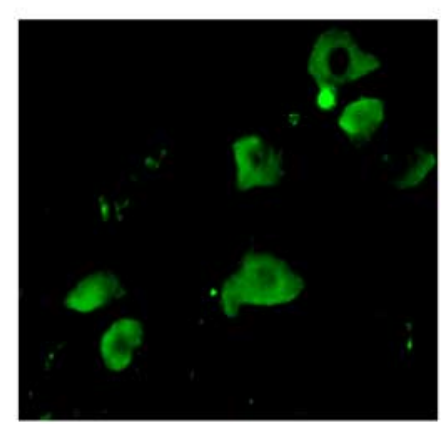

Spinal cord (hrGFP)
$B_{\text {(i) }}$

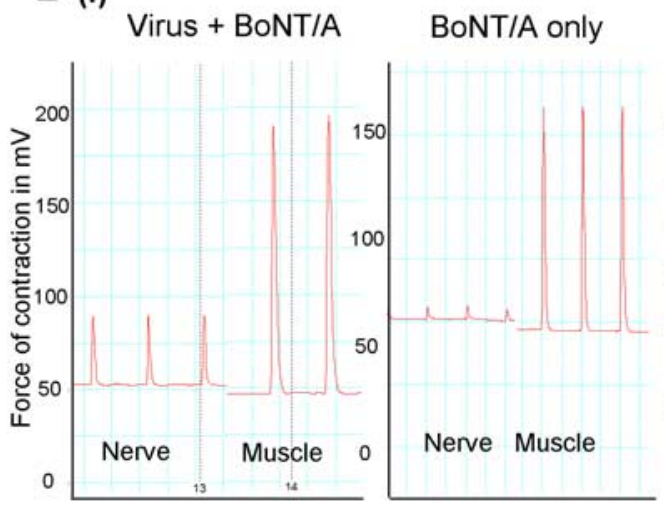

$1 \mathrm{~Hz}$ (ii) Virus + BoNT/A

BoNT/A only

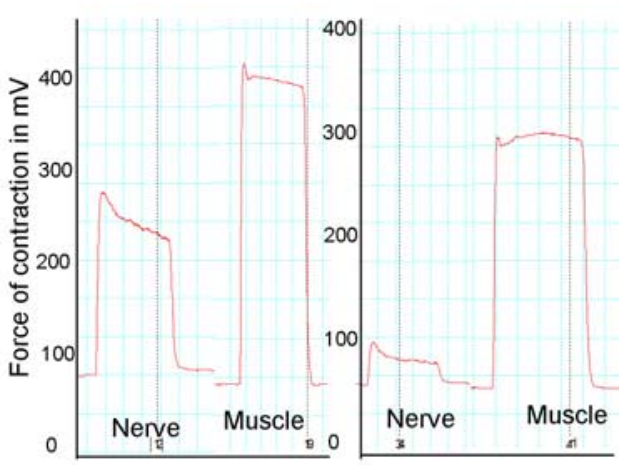

$60 \mathrm{~Hz}$

Figure 4. Peripheral administration of therapeutic AAVs results in their retrograde transport and partial protection against subsequently administered BoNT/A. $A$, Fluorescent microscopic detection of hrGFP in rat spinal lumbar motor neurons after peripheral administration of AAV-hrGFP. B, Rats were injected three times with $50 \mu$ of $10^{11} \mathrm{GCS} / \mathrm{ml} \mathrm{AAV}$-His ${ }_{6}$-S25-R198T into the right soleus muscle; after $7 \mathrm{~d}, \sim 2 \times \mathrm{LD}_{50}$ of BoNT/A was injected bilaterally. Single (i) and tetanic (ii) stimulation from best-responding rats show the improvement resulting from viral preinjection.

terminal sprouts (Pestronk and Drachman, 1978; Brown et al., 1980)], and the duration of paralysis (Meunier et al., 2003). The data herein revealed a reduction in synaptic remodeling when paralysis is limited by overexpression of cleavage-resistant S25 in nerve endings, substantiating the hypothesis that the extent of neurotransmission blockade, along with the duration, are strong determinants of synapse remodeling. The changes in sprouting seen after treatment with the same dose of BoNT/A toxin in the presence or absence of viral protection seem to confirm that the differential effects on sprouting seen previously with the different toxin serotypes are a property of the duration of paralysis and not of the substrate cleaved.

This study attained an important milestone in devising a peripherally deliverable gene therapy that can counteract the prolonged paralysis by BoNT serotypes. The proof of principle obtained provides an encouraging foundation on which to build more versatile vectors based on lentiviruses that could allow the simultaneous transfection of neurons with multiple-toxinresistant S25, VAMP-2, and syntaxin for combating several serotypes and even novel chimera. Furthermore, botulinization solves problems associated with using murine models of human motor neuron diseases. The ability to control the extent/severity of "disease" combined with the availability of a sensitive assay for measuring peripheral neurotransmission and knowledge of the gene required to protect against the toxin's effects, ought to allow meaningful assessment of successful delivery. Such elimination of these multiple variables should enable a more focused and successful optimization of a peripherally administered viral vector that can target therapeutic genes into motor neurons.

\section{References}

Adler M, Keller JE, Sheridan RE, Deshpande SS (2001) Persistence of botulinum neurotoxin A demonstrated by sequential administration of serotypes A and E in rat EDL muscle. Toxicon 39:233-243.

Blasi J, Binz T, Yamasaki S, Link E, Niemann H, Jahn R (1994) Inhibition of neurotransmitter release by clostridial neurotoxins correlates with specific proteolysis of synaptosomal proteins. J Physiol (Paris) 88:235-241.

Brown MC, Holland RL, Ironton R (1980) Nodal and terminal sprouting from motor nerves in fast and slow muscles of the mouse. J Physiol (Lond) 306:493-510.

Bruijn LI, Miller TM, Cleveland DW (2004) Unraveling the mechanisms involved in motor neuron degeneration in ALS. Annu Rev Neurosci 27:723-749.

Burger C, Nash K, Mandel RJ (2005) Recombinant adeno-associated viral vectors in the nervous system. Hum Gene Ther 16:781-791. de Paiva A, Meunier FA, Molgo J, Aoki KR, Dolly JO (1999) Functional repair of motor endplates after botulinum neurotoxin type A poisoning: biphasic switch of synaptic activity between nerve sprouts and their parent terminals. Proc Natl Acad Sci USA 96:3200-3205.

Dolly JO, Aoki KR (2006) The structure and mode of action of different botulinum toxins. Eur J Neurol 13 [Suppl 4]:1-9.

Foran PG, Mohammed N, Lisk GO, Nagwaney S, Lawrence GW, Johnson E, Smith L, Aoki KR, Dolly JO (2003) Evaluation of the therapeutic usefulness of botulinum neurotoxin B, C1, E, and F compared with the long lasting type A. Basis for distinct durations of inhibition of exocytosis in central neurons. J Biol Chem 278:1363-1371.

Kaspar BK, Llado J, Sherkat N, Rothstein JD, Gage FH (2003) Retrograde viral delivery of IGF-1 prolongs survival in a mouse ALS model. Science 301:839-842.

Keller JE, Neale EA, Oyler G, Adler M (1999) Persistence of botulinum neurotoxin action in cultured spinal cord cells. FEBS Lett 456:137-142.

Lawrence GW, Foran P, Dolly JO (1996) Distinct exocytotic responses of intact and permeabilised chromaffin cells after cleavage of the $25-\mathrm{kDa}$ synaptosomal-associated protein (SNAP-25) or synaptobrevin by botulinum toxin A or B. Eur J Biochem 236:877-886.

Mannes AJ, Caudle RM, O'Connell BC, Iadarola MJ (1998) Adenoviral gene transfer to spinal-cord neurons: intrathecal vs. intraparenchymal administration. Brain Res 793:1-6.

Meunier FA, Lisk G, Sesardic D, Dolly JO (2003) Dynamics of motor nerve terminal remodeling unveiled using SNARE-cleaving botulinum toxins: the extent and duration are dictated by the sites of SNAP- 25 truncation. Mol Cell Neurosci 22:454-466.

Nicolopoulos-Stournaras S, Iles JF (1983) Motor neuron columns in the lumbar spinal cord of the rat. J Comp Neurol 217:75-85.

O'Sullivan GA, Mohammed N, Foran PG, Lawrence GW, Oliver Dolly J (1999) Rescue of exocytosis in botulinum toxin A-poisoned chromaffin cells by expression of cleavage-resistant SNAP-25. Identification of the minimal essential C-terminal residues. J Biol Chem 274:36897-36904.

Pestronk A, Drachman DB (1978) Motor nerve sprouting and acetylcholine receptors. Science 199:1223-1225.

Wang P, Yang G, Mosier DR, Chang P, Zaidi T, Gong YD, Zhao NM, Dominguez B, Lee KF, Gan WB, Zheng H (2005) Defective neuromuscular synapses in mice lacking amyloid precursor protein (APP) and APPlike protein 2. J Neurosci 25:1219-1225.

Washbourne P, Cansino V, Mathews JR, Graham M, Burgoyne RD, Wilson MC (2001) Cysteine residues of SNAP-25 are required for SNARE disassembly and exocytosis, but not for membrane targeting. Biochem J 357:625-634.

Zolotukhin S, Byrne BJ, Mason E, Zolotukhin I, Potter M, Chesnut K, Summerford C, Samulski RJ, Muzyczka N (1999) Recombinant adenoassociated virus purification using novel methods improves infectious titer and yield. Gene Ther 6:973-985. 